\section{View the meniscus: MicroCT strategies for enhancing microstructure visualization}

\author{
Gregorio Marchiori, ${ }^{1}$ Melania Maglio, ${ }^{1}$ \\ Lucia Martini, ${ }^{1}$ Andrea Visani, ${ }^{1}$ \\ Stefano Zaffagnini, ${ }^{2}$ Milena Fini ${ }^{1}$
}

1IRCCS Istituto Ortopedico Rizzoli, SC Technological and Surgical Sciences, Bologna; 2IRCCS Istituto Ortopedico Rizzoli, II Clinic, Bologna, Italy

\begin{abstract}
The optimization of imaging protocols for soft tissue visualization is mandatory in the musculoskeletal field. Combining these techniques with histology and mechanics can provide a comprehensive structural and functional assessment of the tissue.
\end{abstract}

\section{Introduction}

Menisci play a key role in the knee joint, being involved in mechanical load distribution, shock absorption, proprioception etc. Meniscal injuries, degenerative or traumatic, can worsen up to subchondral bone lesions, leading to painful, debilitating symptoms, requiring surgical treatments. The advancement in the ex vivo models for the study of structural characteristics of

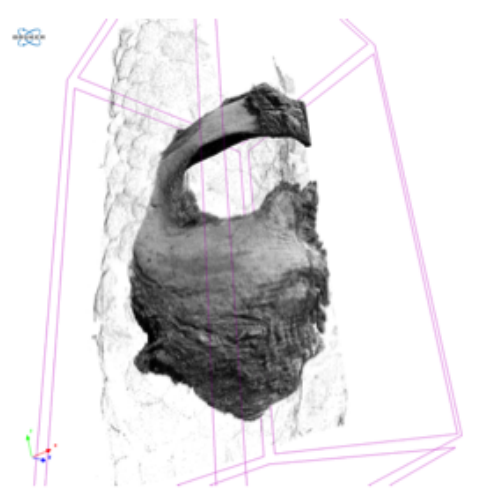

Figure 1. 3D visualization of dried meniscus. meniscus finds a relevant blank in the validation of imaging techniques for the tridimensional visualization of meniscus microstructure, still poorly explored in terms of possible alterations of the microstructure itself that would compromise the analysis. Optimizing imaging methods for visualizing the structure of soft tissues is an indispensable tool for the improvement of diagnostic capabilities and for the development of artificial substitutes to be used in combination with surgical approaches, and is the objective of this study with focus on meniscus.

\section{Materials and Methods}

Human lateral menisci, harvested from patients undergoing total knee arthroplasty, were scanned using microcomputer tomography (microCT) Skyscan 1172 (Bruker, Belgium). Menisci were scanned either immediately after formalin fixation or after undergoing a drying procedure, using increasing alcohol concentrations and final treatment with hexamethyldisilazane. ${ }^{1}$

\section{Results}

The comparison of microtomographical images obtained from not dryed/dryed samples showed that the drying procedure allows a clearer microstructure identification (Figure 1). Histological analyses for the comparative evaluations of preservation of the morphological characteristics of the sample after the application of contrast protocols are in progress.

\section{Discussion and Conclusions}

The possibility to use human biological tissue as waste material from surgical room represents a great advantage, being able to avoid the use of animal models in preliminary evaluation phases, such as in advanced imaging of natural or engineered meniscus microstructure, focus of this study. Such advances can allow more accurate and reliable evaluations, and to select, based on the results obtained, the best evaluated options in order to optimize and refine eventual pre-
Correspondence:Gregorio Marchiori, IRCCS Istituto Ortopedico Rizzoli, SC Technological and Surgical Sciences, Bologna, Italy.

E-mail: gregorio.marchiori@ior.it

Key words: MicroCT; meniscus; contrast agent.

Acknowledgments: The work is supported by Ministry of Health $(5 \times 1000$ project year 2019: "Modellazione biomeccanica del gesto motorio per garantire il ritorno alla attività sportiva dopo ricostruzione del legamento crociato anteriore" and "Medicina rigenerativa e riparativa personalizzata per le patologie dei tessuti muscolo-scheletrici e la chirurgia ricostruttiva ortopedica").

Disclosures: The authors declare no conflict of interest.

Conference presentation: This paper was presented at the Third Centro 3R Annual Meeting - L'era delle 3R: modelli in silico, in vitro e in vivo per promuovere la ricerca traslazionale 30 September - 1 October 2021, Evento online organizzato dal Politecnico di Torino.

Received for publication: 9 July 2021.

Accepted for publication: 7 September 2021.

This work is licensed under a Creative Commons Attribution NonCommercial 4.0 License (CC BY-NC 4.0).

(C) Copyright: the Author(s), 2021

Licensee PAGEPress, Italy

Biomedical Science and Engineering 2021; 4(s1):180 doi:10.4081/bse.2021.180

clinical in vivo models for successive feasibility and efficacy assessment.

Further developments can evaluate the preservation of mechanical, i.e. functional, properties and biological features of the tissue after applying contrast protocols.

\section{References}

1. Karjalainen VP, Kestilä I, Finnilä MA, et al. Quantitative three-dimensional collagen orientation analysis of human meniscus posterior horn in health and osteoarthritis using micro-computed tomography. Osteoarthritis Cartilage 2021;29:762-72. 